\title{
Cyclometalated Iridium-Coumarin Ratiometric Oxygen Sensors: Improved Signal Resolution and Tunable Dynamic Ranges
}

Received 00th January 20xx, Accepted 00th January 20xx DOI: $10.1039 / \times 0 \times x 00000 x$

\begin{abstract}
Yanyu Wu‡, Gregory D. Sutton¥, Michael D. S. Halamicek, and Thomas S. Teets*
In this work we introduce a new series of ratiometric oxygen sensors for hypoxic environments based on phosphorescent cyclometalated iridium centers partnered with organic coumarin fluorophores. Three different cyclometalating ligands and two different pyridyl-containing coumarin types were used to prepare six target complexes with tunable excited-state energies. Some of the complexes exhibit only phosphorescence originating from the cyclometalated Ir moiety, as a result of excited-state energy transfer from the coumarin to the Ir-centered excited states. Three of the complexes display dual emission, with fluorescence arising from the coumarin ligands and phosphorescence from the cyclometalated iridium synthons, and hence function as ratiometric oxygen sensors. Oxygen quenching experiments with these complexes demonstrate that the iridium centered phosphorescence is quenched under $\mathrm{O}_{2}$ while fluorescence is unaffected. These sensors have good signal resolution, and the sensitivity and dynamic range, measured with Stern-Volmer analysis, span two orders of magnitude. This work demonstrates that this simple, modular approach for conjoining fluorescent and phosphorescent molecules can produce effective oxygen sensors with a wide range of attributes.
\end{abstract}

\section{Introduction}

Chemical and biochemical mechanisms of aerobic metabolism largely depend on molecular oxygen. ${ }^{1-3}$ Tumor hypoxia is associated with a variety of common diseases ${ }^{4}$ and therefore the sensing of triplet oxygen has been a recent area of interest. ${ }^{5-10}$ The level of molecular oxygen in tumors is a good indicator of metabolic state and can help guide therapy for cancer treatment. Beyond applications in pathology, accurate oxygen sensing with good spatial and temporal resolution is critical for monitoring physiological responses to extreme environments, e.g. subsea, arctic, and outer space. ${ }^{11}$ Luminescent sensing of oxygen is particularly appealing, where the oxygen concentration is analyzed via the color, intensity, and/or lifetime of photoluminescence. In principle any phosphorescent compound can serve as an oxygen sensor, since molecular triplet states are efficiently quenched by $\mathrm{O}_{2} \cdot{ }^{12}$ However, phosphorescent compounds give a "turn-off" response to oxygen, so their use requires accurate measurement of absolute luminescence intensity, which is prone to issues with calibration and reproducibility, resulting from variable excitation power, optical path length, and in biological applications heterogeneous cellular environments. ${ }^{13}$ Measurement of the phosphorescence lifetime attenuation works well in principle for sensing oxygen but is more technically complex. Luminescent ratiometric oxygen sensors avoid these limitations by incorporating two emission signals which are differentially modulated by $\mathrm{O}_{2}$, and hence the ratio of the two emission intensities can provide a simple and accurate readout of oxygen concentration.

Classical ratiometric oxygen sensors combine a fluorescent moiety with a molecular phosphor. These constructs have quenched phosphorescence in the presence of $\mathrm{O}_{2}$ while

University of Houston, Department of Chemistry, 3585 Cullen Blvd., Room 112, Houston, TX 77204-5003, USA. Email: tteets@uh.edu.

+ Electronic Supplementary Information (ESI) available: Experimental details, X-ray crystallography details, NMR spectra, additional UV-vis absorption, excitation, and photoluminescence spectra. See DOI: 10.1039/x0xx00000x

$¥$ These authors contributed equally. fluorescence is unaffected. Previous designs of ratiometric $\mathrm{O}_{2}$ sensors include quantum dots with phosphorescent metal complexes tethered to the surface ${ }^{14}$ and metal-organic frameworks (MOFs) that combine fluorescent and phosphorescent components. ${ }^{15,16}$ Polymer nanocomposites are also quite common as ratiometric oxygen sensors; these include polymer beads embedded with both molecular phosphors and fluorophores, ${ }^{17-20}$ fluorescent polymers with covalently attached or physically blended phosphorescent molecules, ${ }^{21,22}$ and TADF copolymers where the ratio of prompt to delayed fluorescence is used to measure oxygen. ${ }^{23}$ There are also singlecomponent compounds with dual emission and ratiometric oxygen response, such as bimetallic lanthanide complexes from metal-centered excited states ${ }^{24}$ and boron clusters ${ }^{25}$ or lutetium porphyrin compounds ${ }^{26}$ with dual fluorescence/phosphorescence emission.

Among potential phosphorescent molecules for ratiometric sensing applications, cyclometalated iridium complexes, which have dominated the electroluminescence field, ${ }^{27,28}$ have perhaps the most desirable attributes. They are chemically robust, can be engineered to luminesce in any part of the visible or near-infrared spectrum, often with high quantum yields, and their phosphorescence lifetimes (on the order of microseconds) are well-suited for detecting typical atmospheric or physiological concentrations of $\mathrm{O}_{2}$. Nevertheless, only a few previously reported ratiometric oxygen sensors included cyclometalated iridium complexes. Cyclometalated iridium complexes have been linked to conjugated polymers which then function as ratiometric oxygen sensors, ${ }^{29}$ and most relevant to the present work, red-phosphorescent cyclometalated iridium complexes have been paired with blue-fluorescent coumarins to access biocompatible ratiometric $\mathrm{O}_{2}$ sensors. ${ }^{5,30}$ This latter design is effective, although the synthetic strategy involves upwards of 12 steps, owing primarily to the complex polyproline-substituted acetylacetonate spacer between the iridium center and the coumarin.

One of our primary goals in this area of research has been to design simpler, more generalizable synthetic strategies for preparing ratiometric oxygen sensors featuring cyclometalated iridium. In doing so, it would be much easier to modify the sensor attributes and optimize them for a specific application. 
The spectral profile, the resolution between the phosphorescence and fluorescence signals, the photoluminescence quantum yield, and the triplet lifetime, which is the critical determinant of the oxygen sensing dynamic range, can all in principle be quickly modified. Our initial foray into this area presented a series of bis-cyclometalated iridium complexes joined with pyridyl-substituted BODIPY fluorophores. ${ }^{31}$ The complexes are prepared in a few simple synthetic steps, the last one involving the generation of a substitutionally labile cyclometalated iridium intermediate that "snaps" together with the pyridyl-substituted BODIPY under mild conditions. This same synthetic strategy has been used in our group to access other multi-component photoactive structures, which are not ratiometric sensors but nonetheless are valuable platforms for studying excited-state energy transfer pathways. ${ }^{32,33}$ Some of the Ir-BODIPY compounds prepared in this way functioned as effective ratiometric oxygen sensors, with dynamic ranges that spanned hypoxic levels of $\mathrm{O}_{2}$ $\left(p \mathrm{O}_{2} \leq 150 \mathrm{mmHg}\right) \cdot{ }^{31}$

In this work, we present two significant advances in the design of ratiometric oxygen sensors featuring cyclometalated iridium, showing that the simple synthetic approach we have developed facilitates modification and optimization of the sensor attributes. One limitation of our first-generation IrBODIPY oxygen sensors is a significant overlap between the BODIPY's green fluorescence and the broad red phosphorescence from the iridium center. This poor signal resolution between the two luminescence channels means the effective ratiometric response never reaches zero, limiting the dynamic range. Here we study complexes that replace the green-fluorescent BODIPY with a blue-fluorescent coumarin, and in one such complex we also shift the phosphorescence deeper to the red, achieving greatly improved signal resolution showing no overlap between fluorescence and phosphorescence. This improved signal resolution contributes to a much larger dynamic range for oxygen sensing. A second major discovery in this work, enabled by the ability to quickly "mix and match" organic fluorophores and bis-cyclometalated iridium phosphors, is the preparation of sensors that are sensitive to much lower concentrations of $\mathrm{O}_{2}$. For certain applications it would be desirable to have sensors that show large responses to very low levels of oxygen as opposed to a gradual response over a wider range, and in this work, we show that is possible with some of our coumarin-based sensors. In total, this paper describes the preparation and characterization of six new cyclometalated iridium-coumarin complexes, and the three that show dual luminescence are subjected to an in-depth quantification of their oxygen-sensing attributes in abiological solutions.

\section{Results and Discussion}

\section{Synthesis of Ir-Coumarin Complexes}

The general synthetic preparation of the complexes is outlined in Scheme 1, and in most cases follows closely with the procedure used to prepare our first-generation Ir-BODIPY sensors. ${ }^{31}$ Three precursor classes were used to prepare the six compounds in this study. Two pyridyl-substituted coumarin compounds, abbreviated $\mathbf{C}-\mathbf{1}$ and $\mathbf{C}-\mathbf{2}$, were synthesized using known procedures. ${ }^{34,35}$ Chloro-bridged cyclometalated iridium dimers $\left[\operatorname{Ir}\left(\mathrm{C}^{\wedge} \mathrm{N}\right)_{2}(\mu-\mathrm{Cl})\right]_{2}\left(\mathbf{1} \mathbf{a}-\mathbf{c} \mathrm{C}^{\wedge} \mathrm{N}=\right.$ cyclometalating ligand $)$ are ubiquitous precursors accessed by the method of Nonoyama. ${ }^{36}$ In all iridium complexes described here, the letter in the numerical abbreviation denotes the cyclometalating ligand

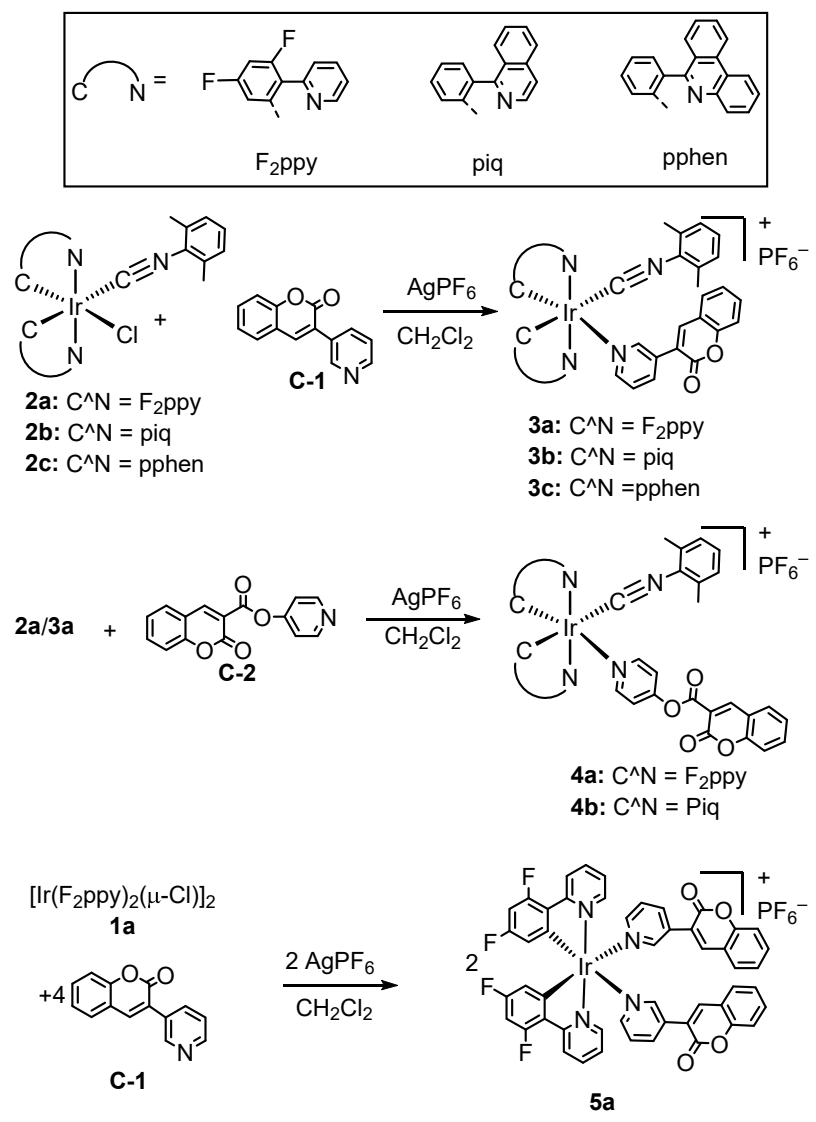

Scheme 1. Synthesis of cyclometalated iridium-coumarin compounds.

used, 2-(2,4-difluorophenyl)pyridine $\left(F_{2}\right.$ ppy, a), 1phenylisoquinoline (piq, b), and 6-phenylphenanthridine (pphen, c), which normally produce blue, red, and deep red phosphorescence when chelated to iridium(III), respectively. The bis-cyclometalated iridium precursors $\mathbf{2} \mathbf{a}-\mathbf{c}$ of the type $\operatorname{Ir}\left(\mathrm{C}^{\wedge} \mathrm{N}\right)_{2}\left(\mathrm{CNAr}{ }^{\mathrm{dmp}}\right)(\mathrm{Cl}) \quad\left(\mathrm{CNAr}{ }^{\mathrm{dmp}}=2,6-\right.$ dimethylphenylisocyanide) were synthesized as previously described by our group, cleaving the chloro-bridged dimers with isocyanides. ${ }^{32,37}$ Finally, the cyclometalated iridium-coumarin dyads $\mathbf{3} \mathbf{a}-\mathbf{c}$ and $\mathbf{4} \mathbf{a}-\mathbf{b}$ were prepared using a simple, one-pot reactions between the respective coumarin and the isocyanide precursors $\mathbf{2 a - c}$ in the presence of $\mathrm{AgPF}_{6}$. Here we also introduce a new structural class where the bis-cyclometalated iridium center is coordinated to two pyridyl-coumarins, represented by complex $\mathbf{5 a}$. This latter compound is prepared directly from the chloro-bridged cyclometalated iridium dimer 1a and coumarin $\mathbf{C}-\mathbf{1}$, also in the presence of $\mathrm{AgPF}_{6}$. These reactions are reasonably high-yielding based on crude NMR spectra but following multiple rounds of rigorous purification low to moderate isolated yields (20-60\%) were obtained. ${ }^{1} \mathrm{H}$ and 
${ }^{19} \mathrm{~F}$ NMR spectroscopy was performed on all the target complexes to affirm their identity and bulk purity, and ${ }^{13} \mathrm{C}\left\{{ }^{1} \mathrm{H}\right\}$ NMR was also collected on complexes $\mathbf{3 b}, \mathbf{3 c}$ and $\mathbf{4 b}$ which lack fluorination in the $C^{\wedge} N$ ligands. ${ }^{19} \mathrm{~F} N M R$ spectra were particularly useful for determining the $C_{1}$ point group symmetry of $\mathbf{3 a}$ and $\mathbf{4 a}$ and the $C_{2}$ symmetry of $\mathbf{5 a}$; the former show four distinct ${ }^{19} \mathrm{~F}$ resonances for the $\mathrm{F}_{2}$ ppy ligands, whereas the latter only shows two. The NMR spectra of the complexes can be found in Fig. S1-S15 in the ESI. +

Single-crystal $X$-ray diffraction further confirms the molecular structures of the three $F_{2}$ ppy complexes $3 a-5 a, \S$ which are shown in Fig. 1. Refinement and diffraction data for

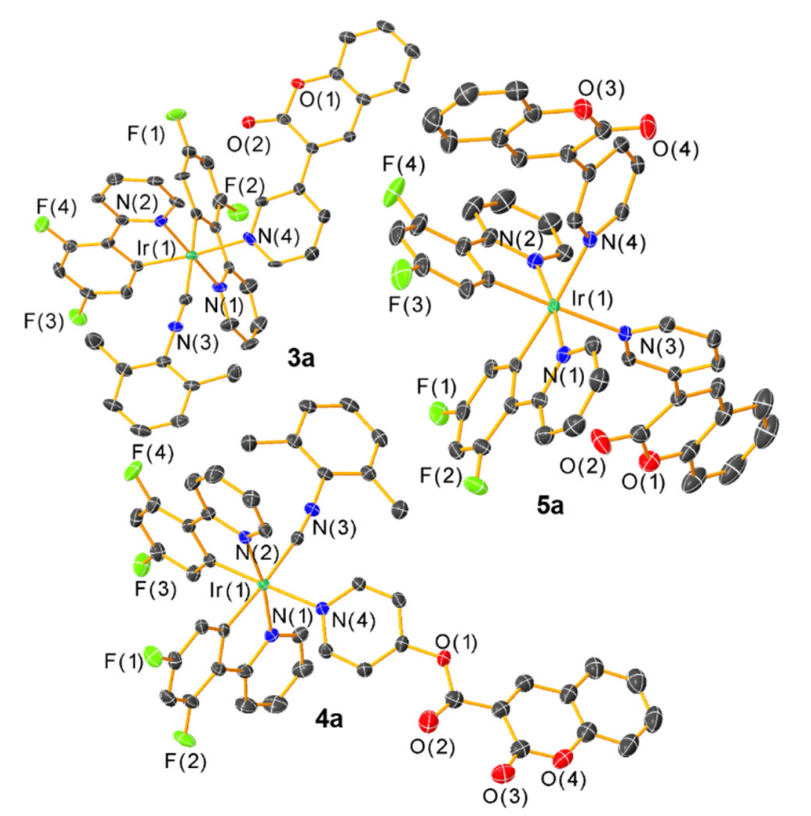

Fig. 1. Molecular structures of complexes $\mathbf{3 a - 5 a}$, determined by single-crystal X-ray diffraction. Ellipsoids are drawn at the $50 \%$ probability level with hydrogen atoms, solvent molecules and counterions omitted.

these three complexes are summarized in Table S1 of the ESI. All the complexes show a distorted octahedral geometry centered on the Ir atom. The pyridyl nitrogen atoms from the $F_{2}$ ppy ligands are in a trans orientation, with the other two cisoriented coordination sites are occupied by one $\mathrm{CN}^{\mathrm{dmp}}$ and one pyridyl coumarin ligand for complexes $3 \mathbf{a}$ and $4 \mathbf{a}$, and two pyridyl coumarins in $\mathbf{5 a}$. The $C_{1}$ symmetry of $\mathbf{3 a}$ and $4 \mathbf{a}$ and the approximate $C_{2}$ symmetry of $\mathbf{5 a}$, readily apparent from their NMR spectra, are confirmed by the crystal structures. The Ir center is covalently bound to the coumarin via the $\mathrm{N}$ atom in the pyridyl moiety, with Ir-N bond distances ranging between 2.172 and $2.190 \AA$, slightly longer than the $\mathrm{I}-\mathrm{N}$ bond distances between the $F_{2}$ ppy nitrogen atom and the iridium center, which range from 2.046 to $2.079 \AA$.

\section{Photophysical Properties}

Fig. S16 and S17 of the ESIt display the overlaid UV-vis absorption and photoluminescence emission spectra of free coumarins C-1 and C-2. The absorption spectra of both coumarins show two near-UV absorption peaks between 300$350 \mathrm{~nm}$, which can be assigned to $S_{0} \rightarrow S_{1}$ and $S_{0} \rightarrow S_{2}$ transitions. Both C-1 and C-2 display deep-blue fluorescence emission with maxima at $\lambda=406 \mathrm{~nm}$ and $420 \mathrm{~nm}$, respectively, with moderate Stokes shifts of $6100 \mathrm{~cm}^{-1}$ for both compounds.

Fig. 2 shows overlaid UV-vis absorption and photoluminescence spectra of each iridium-coumarin compound, with the numerical data for the photoluminescence summarized in Table 1. The iridium-coumarin complexes have near-UV absorption in the same regions as free coumarins C-1 and C-2, overlapped with other strong absorption bands that are likely $\pi \rightarrow \pi^{*}$ transitions from the $C^{\wedge} N$ ligands chelated to iridium. Metal-to-ligand charge transfer (MLCT) bands originating from an $\operatorname{Ir}(5 d) \rightarrow C^{\wedge} N\left(\pi^{*}\right)$ transitions are clearly observed in the complexes with the more conjugated $\mathrm{C}^{\wedge} \mathrm{N}$ ligands piq and pphen. These broad bands occur at ca. $410 \mathrm{~nm}$ in piq complexes $\mathbf{3 b}$ and $\mathbf{4 b}$ and ca. $420 \mathrm{~nm}$ in pphen complex 3c.

Photoluminescence spectra of all cyclometalated Ir coumarin complexes were recorded both at room temperature in $\mathrm{CH}_{2} \mathrm{Cl}_{2}$ and at $77 \mathrm{~K}$ in $\mathrm{CH}_{2} \mathrm{Cl}_{2}$ /toluene (1:3) glass, which are also

Table 1. Summary of photoluminescence properties of the iridium-coumarin complexes. Room-temperature photoluminescence spectra were measured in $\mathrm{CH}_{2} \mathrm{Cl}_{2}$ at $293 \mathrm{~K}$. Photoluminescence at $77 \mathrm{~K}$ were obtained in a mixture of $\mathrm{CH}_{2} \mathrm{Cl}_{2} /$ toluene $(1: 3 \mathrm{v} / \mathrm{v})$. Samples for steady-state photoluminescence measurement were excited at $310 \mathrm{~nm}$, and for lifetime measurements at $330 \mathrm{~nm}$.

\begin{tabular}{|c|c|c|c|c|}
\hline & $\begin{array}{l}\lambda_{\mathrm{em}} / \mathrm{nm} \\
(293 \mathrm{~K})\end{array}$ & $\begin{array}{c}\lambda_{\text {em }} / \mathrm{nm} \\
(77 \mathrm{~K})\end{array}$ & $\Phi_{\mathrm{PL}}$ & $\tau / \mu \mathrm{s}$ \\
\hline $3 a$ & $\begin{array}{c}395,447,477 \\
547,592,648\end{array}$ & $\begin{array}{c}396,443,467 \\
525,567,613\end{array}$ & N.D. & N.D. \\
\hline 3b & 587,627 & $576,624,677$ & 0.17 & 6.4 \\
\hline $3 c$ & 413,650 & $406,614,655$ & $0.022^{\mathrm{a}}, 0.086^{\mathrm{b}}$ & 2.7 \\
\hline $4 a$ & $\begin{array}{c}415,448,478 \\
506\end{array}$ & $\begin{array}{c}407,443,489 \\
516,556\end{array}$ & 0.027 & 1.6 \\
\hline $4 b$ & 591,626 & $577,625,679$ & 0.22 & 6.5 \\
\hline $5 a$ & $\begin{array}{c}407,550,592 \\
650\end{array}$ & $\begin{array}{r}406,444,475 \\
521,565,614\end{array}$ & $0.0061^{a}, 0.016^{b}$ & N.D. \\
\hline
\end{tabular}

a Fluorescence quantum yield. ${ }^{\mathrm{b}}$ Phosphorescence quantum yield. 

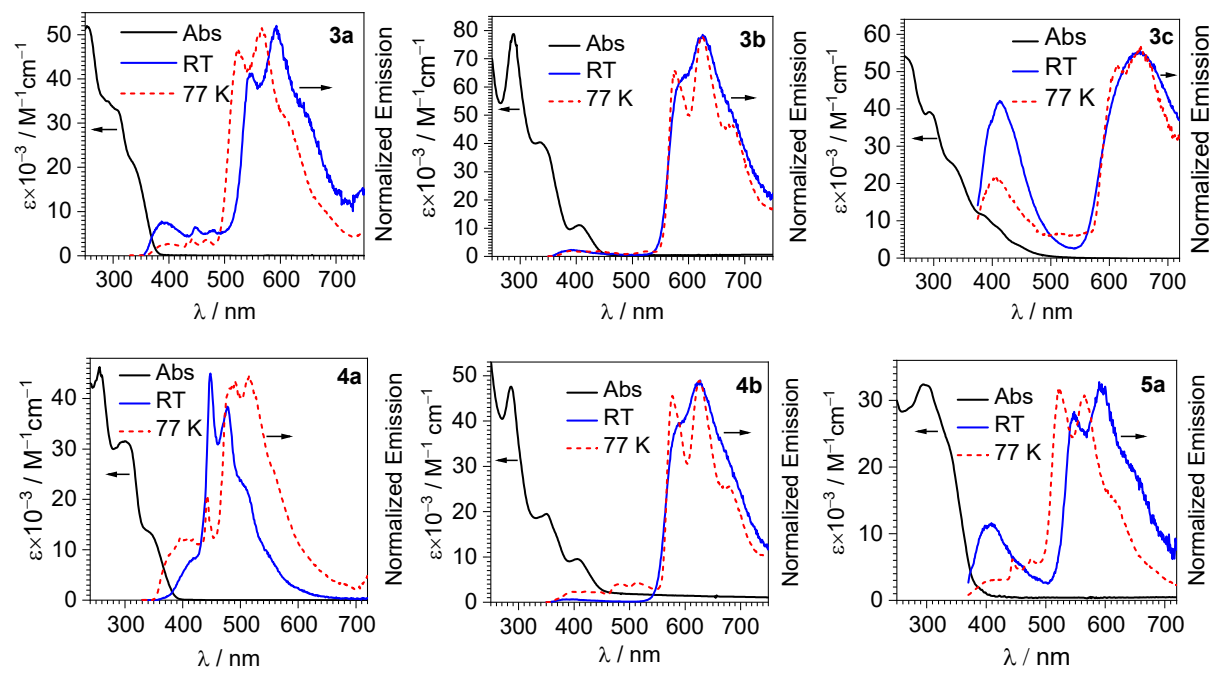

Fig. 2. Overlaid UV-vis absorption and photoluminescence spectra of the iridium-coumarin complexes. UV-vis absorption (solid black line) and room-temperature photoluminescence (blue solid line) were both recorded in $\mathrm{CH}_{2} \mathrm{Cl}_{2}$ at $293 \mathrm{~K}$, and photoluminescence at $77 \mathrm{~K}$ (red dashed line) was measured in $1: 3(\mathrm{v} / \mathrm{v}) \mathrm{CH}_{2} \mathrm{Cl}_{2} /$ toluene.

presented in Fig. 2. Among the six compounds, there are three distinct patterns for the room-temperature photoluminescence:

1) In piq complexes $\mathbf{3} \mathbf{b}$ and $\mathbf{4} \mathbf{b}$, the only appreciable photoluminescence is phosphorescence from the biscyclometalated iridium fragment. The emission both at 298 and $77 \mathrm{~K}$ is almost identical to the previously described model complex $\left[\operatorname{Ir}(\text { piq })_{2}\left(\mathrm{CNAr}^{\mathrm{dmp}}\right)\right.$ (pyridine) $]\left(\mathrm{PF}_{6}\right) .^{32}$ The lifetimes of $\mathbf{3 b}$ and $4 \mathbf{b}$, c.a. $6 \mu \mathrm{s}$, are likewise similar to the model complex, and photoluminescence quantum yields $\left(\Phi_{\mathrm{PL}}\right)$ are about a factor of two higher, 0.17 (3b) and 0.22 (4b), vs. 0.096 in the model complex. Because these two compounds only exhibit phosphorescence and no fluorescence, they are not suitable candidates for ratiometric oxygen sensing.

2) In complexes $\mathbf{3 c}$ and $\mathbf{4 a}$, there is dual emission involving fluorescence from the coumarin and phosphorescence from the $\left[\operatorname{Ir}\left(\mathrm{C}^{\wedge} N\right)_{2}\right]^{+}$moiety. The fluorescence occurs at similar wavelengths as the free coumarins (see Fig. S16 and S17), and the phosphorescence is dictated by the cyclometalating ligand. In $4 a$ the coumarin fluorescence overlaps strongly with the phosphorescence, appearing as a shoulder on the high-energy side of the phosphorescence vibronic progression. This substantial overlap between fluorescence and phosphorescence means that 4 a would not function well as a ratiometric sensor. The phosphorescence spectrum and lifetime (1.6 $\mu \mathrm{s})$ in 4 a strongly resemble those of an $\left[\operatorname{Ir}\left(\mathrm{F}_{2} \mathrm{ppy}\right)_{2}\left(\mathrm{CNAr}{ }^{\mathrm{dmp}}\right)\right.$ (pyridine) $]\left(\mathrm{PF}_{6}\right)$ model complex, ${ }^{32}$ further confirming this assignment. In pphen complex 3c, the blue fluorescence from the coumarin $\left(\lambda_{\max }=413 \mathrm{~nm}\right)$ is well-separated from the deep red phosphorescence $\left(\lambda_{\max }=650 \mathrm{~nm}\right)$, with no appreciable overlap between the two signals. The good resolution between the two signals allowed us to separately integrate the fluorescence and phosphorescence bands and determine quantum yields for each. With excitation at $310 \mathrm{~nm}$, the fluorescence quantum yield is 0.022, and the phosphorescence quantum yield is 0.086 . The large separation between the two bands also makes complex $\mathbf{3 c}$ ideally suited for ratiometric sensing, as described below.

3) In the other two $F_{2}$ ppy complexes $\mathbf{3 a}$ and $\mathbf{5 a}$, dual luminescence with some blue fluorescence from the coumarin is likewise observed. However, the phosphorescence in these compounds, with two vibronic maxima at ca. 550 and $590 \mathrm{~nm}$, occurs at much longer wavelength than typically observed in $\left[\operatorname{Ir}\left(\mathrm{F}_{2} \mathrm{ppy}\right)_{2}\right]^{+}$complexes. This structured luminescence in $\mathbf{3 a}$ and $\mathbf{5 a}$ is ascribed to phosphorescence originating from a coumarin-centered triplet state and is similar to the luminescence observed in iridium complexes with coumarin-based cyclometalating ligands. ${ }^{38,39}$ In these compounds the $\left[\operatorname{Ir}\left(\mathrm{F}_{2} \text { ppy }\right)_{2}\right]^{+}$ triplet state is higher in energy than the coumarincentered state, resulting in population of this lowestenergy state prior to any phosphorescence occurring.

Overlaid UV-vis absorption and excitation spectra of each complex are shown in Fig. S18-S23 of the ESIt, and they reveal additional insights into the excited-state dynamics. Stated briefly, to observe coumarin fluorescence (all but $\mathbf{3 b}$ and $\mathbf{4 b}$ ), the coumarin moiety must be directly excited. Excitation anywhere in the UV-vis absorption window gives rise to phosphorescence, and when monitoring the excitation spectrum at the phosphorescence maximum the UV-vis absorption and excitation spectra match well. In other words, for the $T_{1}$ state Kasha's rule is followed. One interesting observation noted above is that in $F_{2}$ ppy complexes $\mathbf{3 a}$ and $\mathbf{5 a}$, which both use the 3-pyridyl-substituted coumarin, phosphorescence occurs from the coumarin triplet state, whereas in $\mathbf{4 a}$, where the longer carboxy-pyridine linker is used, phosphorescence occurs from the iridium center. This phenomenon underscores the importance of the linker 
between the fluorophore and phosphor in determining the energy-transfer dynamics and suggests that with the longer linker in $\mathbf{4 a}$ triplet energy transfer to the coumarin is not as rapid, and all phosphorescence occurs from the iridium center. Ratiometric Oxygen Sensing

$\mathrm{F}_{2}$ ppy complexes $\mathbf{3} \mathbf{a}$ and $\mathbf{5} \mathbf{a}$ and pphen complex $\mathbf{3} \mathbf{c}$ exhibit clear dual emission and hence were chosen for ratiometric oxygen sensing. A qualitative assessment of all six complexes' response to oxygen was performed by preparing each sample in a nitrogen-filled glovebox, the photoluminescence spectra observed, then the spectra taken again after equilibration in air. The spectra of each complex in $\mathrm{N}_{2}$-saturated versus aerated environments can be found in Fig. S24-S29 of the ESI. + For the dual-emitting compounds there is no change in coumarin fluorescence upon exposure to air, while in all compounds, phosphorescence is quenched. A quantitative assessment was performed on dual-emitting compounds $\mathbf{3 a}, \mathbf{5} \mathbf{a}$ and $\mathbf{3} \mathbf{c}$ by taking photoluminescence spectra at increasing $p \mathrm{O}_{2}$ levels, either until phosphorescence was completely quenched or until atmospheric levels of oxygen (ca. $160 \mathrm{mmHg}$ ) were present. Fig. 3 (left column) shows the spectral change for each complex with increasing $\mathrm{pO}_{2}$ levels, which clearly shows quenching of the longer-wavelength phosphorescence with no change in the coumarin's fluorescence intensity. All three complexes are capable of sensing partial pressures of oxygen below atmospheric content $\left(p \mathrm{O}_{2} \leq 160 \mathrm{mmHg}\right)$, meaning they can be used in hypoxic environments.

A more quantitative description of the oxygen-sensing comes from Stern-Volmer analysis. Equation 1 shows the SternVolmer relationship, where $K_{\mathrm{SV}}$ is the Stern-Volmer constant, $p \mathrm{O}_{2}$ is the oxygen partial pressure, $k_{\mathrm{q}}$ is the quenching rate constant, and $\tau_{0}$ and $\tau$ are the lifetimes without and with oxygen present, respectively. ${ }^{40,41}$ In complexes $\mathbf{3 a}$ and $\mathbf{5 a}$ the photoluminescence is too weak and the lifetimes too long to determine accurate values on our instrumentation, so we analyzed the data with a previously reported modified SternVolmer equation that is appropriate for ratiometric sensors. ${ }^{5}$ In this method $R_{1}$ describes the intensity ratio of phosphorescence to fluorescence, with $R_{l}^{0}$ describing this ratio in the absence of $\mathrm{O}_{2}$. Equation 2 shows this modified Stern-Volmer relationship, using $R_{1}{ }^{0} / R_{1}$ as the dependent variable with $K_{\mathrm{Sv}}$ and $p \mathrm{O}_{2}$ defined the same as in Equation 1. ${ }^{5}$

$$
\begin{gathered}
\frac{\tau_{0}}{\tau}=1+K_{S V} p O_{2}=1+k_{q} \tau_{0} p O_{2} \\
\frac{R_{I}^{0}}{R_{I}}=1+K_{S V} p O_{2}
\end{gathered}
$$

The Stern-Volmer constant $K_{\mathrm{SV}}$ can be obtained from the slope of the linear fit, and all three compounds in Fig. 3 give very different $K_{\mathrm{SV}}$ values, indicating different sensitivities to $\mathrm{O}_{2} . K_{\mathrm{SV}}$ for pphen complex $3 \mathrm{c}$ is $1.3 \times 10^{-2} \mathrm{mmHg}^{-1}$, slightly smaller than
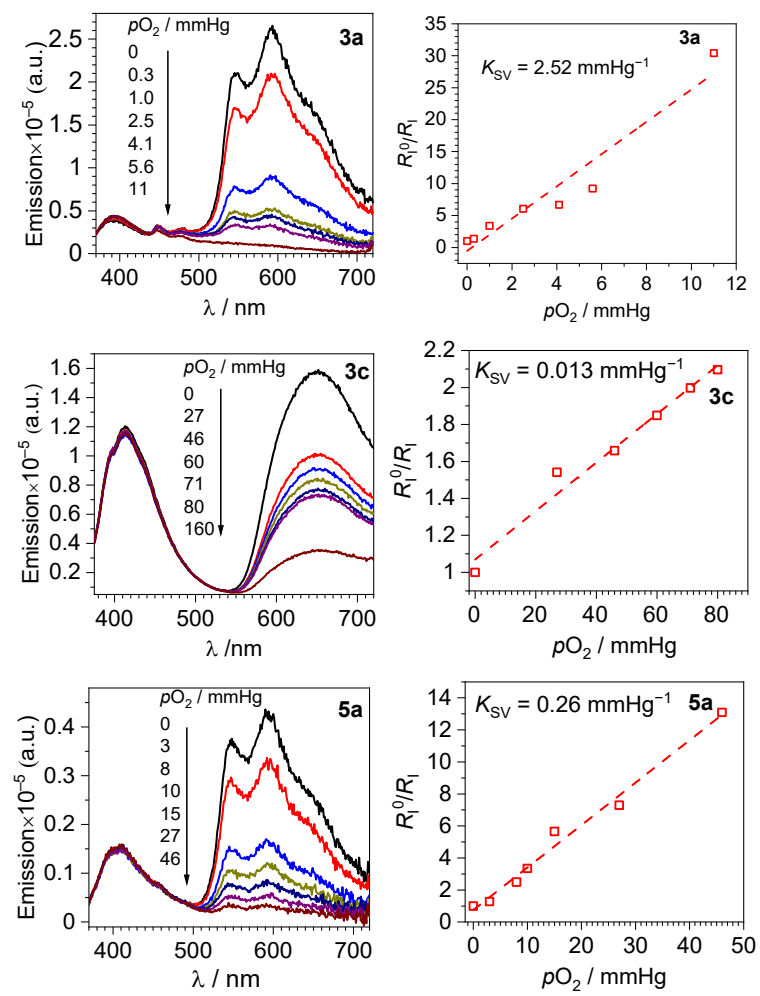

Fig. 3. Spectral response to increasing oxygen concentration (left column), Stern-Volmer oxygen quenching studies (right column) for $\mathbf{3 a}, \mathbf{3} \mathbf{c}$ and $\mathbf{5 a}$. All data were recorded in $\mathrm{CH}_{2} \mathrm{Cl}_{2}$ at $293 \mathrm{~K}$.

those of our first-generation sensors $\left(K_{\mathrm{SV}}=3.0-8.1 \times 10^{-2}\right.$ $\left.\mathrm{mmHg}^{-1}\right),{ }^{31}$ but same order of magnitude. For complexes $3 a$ and 5 a where the phosphorescence arises from the coumarin triplet state, the oxygen sensitivity is much higher, and $K_{\mathrm{SV}}$ is one or two orders of magnitude higher than that of $3 \mathbf{c}$, measured at $0.26(5 \mathrm{a})$ and 2.5 (3a) $\mathrm{mmHg}^{-1}$. For complex $3 \mathrm{c}$ the inherent phosphorescence lifetime $\tau_{0}$ is known $(2.7 \mu \mathrm{s}$, see Table 1$)$, allowing us to use Equation 1 to determine a bimolecular quenching rate constant, $k_{\mathrm{q}}$, of $4.8 \times 10^{3} \mathrm{~s}^{-1} \mathrm{mmHg}^{-1}$, equivalent to $5.2 \times 10^{9} \mathrm{~s}^{-1} \mathrm{M}^{-1}$ using the known solubility of oxygen gas in dichloromethane. ${ }^{42}$ This $k_{\mathrm{q}}$ value approaches the diffusion limit which suggests that $3 \mathrm{c}$ is efficiently quenched by $\mathrm{O}_{2}$, and is within the range of $k_{\mathrm{q}}$ values observed in our first-generation IrBODIPY sensors. ${ }^{31}$ Without available lifetime values we cannot determine $k_{\mathrm{q}}$ values for $\mathbf{3} \mathbf{a}$ and $\mathbf{5} \mathbf{a}$, but in most cases oxygen quenching of triplet states is near the diffusion limit, ${ }^{43}$ so if we assume that $k_{\mathrm{q}}$ spans the range of $10^{9}-10^{10} \mathrm{~s}^{-1} \mathrm{M}^{-1}$, we can estimate that the excited-state lifetime for $\mathbf{3 a}$ is in the range of $10^{-4}$ to $10^{-3} \mathrm{~s}$, and that of $5 \mathrm{a}$ about an order of magnitude shorter in the range of $10^{-5}$ to $10^{-4} \mathrm{~s}$. Both of these are substantially longer than that of $\mathbf{3 c}$, consistent with the organic ${ }^{3}\left(\pi \rightarrow \pi^{*}\right)$ nature of the emissive state in $\mathbf{3 a}$ and $\mathbf{5 a}$, versus the significant $\operatorname{Ir}(5 \mathrm{~d}) \rightarrow \mathrm{C}^{\wedge} \mathrm{N}\left(\pi^{*}\right)^{3} \mathrm{MLCT}$ character in $\mathbf{3 c}$.

To better quantify and compare the dynamic ranges of the sensors, here we define a parameter abbreviated as $p\left(\mathrm{O}_{2}\right)_{90 \%}$, the value of $p\left(\mathrm{O}_{2}\right)$ needed to reach $90 \%$ quenching. In these sensors the good signal resolution allows $R_{1}$ to approach 0 , so $p\left(\mathrm{O}_{2}\right)_{90 \%}$ is defined as the pressure where $R_{1}{ }^{0} / R_{1}=10$. Using the best-fit Stern-Volmer lines in Fig. 3, we extrapolate $p\left(\mathrm{O}_{2}\right)_{90 \%}$ 
values of ca. $4 \mathrm{mmHg}$ (3a), $36 \mathrm{mmHg}$ (5a), and $690 \mathrm{mmHg}$ (3c). Thus, all three sensors operate under very different dynamic ranges, giving good sensitivity to small (3a), intermediate (5a), and large ranges of $\mathrm{pO}_{2}(\mathbf{3 c})$. The slightly smaller $K_{\mathrm{SV}}$ value and much improved signal resolution in $\mathbf{3 c}$ combine to give a much larger dynamic range when compared to our previous Ir-BODIPY sensors, by at least a factor of 10 in terms of the estimated $p\left(\mathrm{O}_{2}\right)_{90 \%}$. The superior signal resolution in $\mathbf{3 c}$ is critical, as the smaller $K_{\mathrm{SV}}$ value accounts for only about a factor of 2.5 difference in dynamic range. All these metrics show that, using our synthetic approach that allows us to easily join different fluorophores and phosphors, we can dial in a wide range of sensor attributes, allowing us to optimize the characteristics for a specific application.

\section{Conclusions}

To summarize, we have synthesized a set of cyclometalated iridium-coumarin dyads applied as ratiometric oxygen sensors. The complexes are prepared using relatively simple synthetic methods, allowing ready access to several structural variants. The compounds have diverse photoluminescence profiles, determined by the choice of cyclometalating ligand on iridium and the linker between the coumarin and the iridium center. In some cases only phosphorescence from the cyclometalated iridium center is observed, and in others dual luminescence involving blue fluorescence from the coumarin and longerwavelength phosphorescence occurs, the latter arising either from an iridium-centered ${ }^{3} \mathrm{MLCT}$ state or a coumarin-centered ${ }^{3}\left(\pi \rightarrow \pi^{*}\right)$ state. Three of the six complexes exhibit well-resolved dual emission, and their oxygen sensing attributes are distinctly improved in relation to our first-generation sensors. In all cases clear resolution between the fluorescence and phosphorescence signals is observed, with no overlap. The sensitivities and dynamic ranges of the sensors span two orders of magnitude, showing that the structural modifications enabled by our synthetic approach can have dramatic consequences on the sensing profile. In complex 3c, where phosphorescence arises from a ${ }^{3} \mathrm{MLCT}$ state centered on the [Ir(pphen $\left.)_{2}\right]^{+}$phosphor, the comparatively small Stern-Volmer constant $\left(K_{\mathrm{SV}}\right)$ and large separation between fluorescence and phosphorescence enables oxygen detection over a wide range of partial pressures, substantially improved over our firstgeneration sensors where the green fluorescence and red phosphorescence overlapped. In compounds $\mathbf{3} \mathbf{a}$ and $\mathbf{5 a}$, the coumarin-centered phosphorescence is apparently much longer lived, leading to much higher sensitivity to low $\mathrm{O}_{2}$ levels. Thus, these sensors are ideally suited to applications where accurate detection of small $\mathrm{O}_{2}$ partial pressures is needed. This work shows the versatility of our approach in designing effective ratiometric oxygen sensors. Future modifications will target improved photoluminescence quantum yields, which are all less than 0.1 in this class of sensors, by leveraging our group's previous insights into the design of red and near-infrared phosphors with high quantum yields. ${ }^{44,45}$

\section{Author Contributions}

Yanyu Wu: conceptualization, formal analysis, investigation, visualization, writing - original draft, writing - review \& editing. Gregory D. Sutton: formal analysis, investigation, visualization, writing - original draft, writing - review and editing. Michael D. S. Halamicek: formal analysis, investigation, writing - review \& editing. Thomas S. Teets: conceptualization, formal analysis, project administration, supervision, visualization, writing review \& editing.

\section{Conflicts of interest}

There are no conflicts to declare.

\section{Acknowledgements}

We thank the University of Houston Grants to Enhance Academic Research (GEAR) and the Welch Foundation (Grant no. E-1887) for funding this research. M.D.S.H. acknowledges the University of Houston Office Undergraduate Research for fellowship support through the SURF and PURS programs.

\section{Notes and references}

$\S 3 a \cdot 0.5 \mathrm{C}_{7} \mathrm{H}_{8}$ : CCDC 2126601, $\mathrm{C}_{48.50} \mathrm{H}_{34} \mathrm{~F}_{10} \mathrm{IrN}_{4} \mathrm{O}_{2} \mathrm{P}, M=1117.96$, Triclinic, $P-1, a=$ $12.373(6) \AA, b=19.541(9) \AA, c=21.137(10) \AA, \alpha=63.579(5)^{\circ}, b=75.787(5)^{\circ}, y=$ $71.958(5)^{\circ}, Z=4,16458$ tot. refln., 16458 ind. refln., $R_{\text {int }}=0.0625, R_{1}=0.045, w R_{2}=$ 0.242. 4a $1.5 \mathrm{C}_{6} \mathrm{H}_{6}:$ CCDC 2126602, $\mathrm{C}_{55} \mathrm{H}_{39} \mathrm{~F}_{10} \mathrm{IrN}_{4} \mathrm{O}_{4} \mathrm{P}, M=1233.07$, Triclinic, $P-1, a=$ 12.0293(3) $\AA, b=13.6426(3) \AA, c=15.8219(3) \AA, \alpha=79.160(1)^{\circ}, b=81.948(1)^{\circ}, v=$ $71.953(1), Z=2,31358$ tot. refln., 9473 ind. refln., $R_{\text {int }}=0.020, R_{1}=0.023, w R_{2}=$ 0.058. 5a: CCDC 2126603, $\mathrm{C}_{50} \mathrm{H}_{30} \mathrm{~F}_{10} \mathrm{IrN}_{4} \mathrm{O}_{4} \mathrm{P}, M=1163.95$, Triclinic, $P-1, a=11.885(2)$ $\AA \AA, b=13.195(3) \AA, c=16.517(3) \AA, \alpha=105.127(2)^{\circ}, b=97.590(2)^{\circ}, y=101.776(2), Z$ $=2,33955$ tot. refln., 11009 ind. refln., $R_{\text {int }}=0.032, R_{1}=0.030, w R_{2}=0.075$.

1 J. Aragonés, P. Fraisl, M. Baes and P. Carmeliet, Cell Metab., 2009, 9, 11-22.

2 J. Pecher and S. Mecking, Chem. Rev., 2010, 110, 6260-6279.

3 C. Wu and D. T. Chiu, Angew. Chem. Int. Ed., 2013, 52, 30863109.

4 M. Höckel and P. Vaupel, J. Natl. Cancer Inst., 2001, 93, 266276.

5 T. Yoshihara, Y. Yamaguchi, M. Hosaka, T. Takeuchi and S. Tobita, Angew. Chem. Int. Ed., 2012, 51, 4148-4151.

6 C. Wu, B. Bull, K. Christensen and J. McNeill, Angew. Chem. Int. Ed Engl., 2009, 48, 2741-2745.

7 S. Zhang, M. Hosaka, T. Yoshihara, K. Negishi, Y. lida, S. Tobita and T. Takeuchi, Cancer Res., 2010, 70, 4490-4498.

8 A. J. Pistner, R. C. Pupillo, G. P. A. Yap, D. A. Lutterman, Y.-Z. Ma and J. Rosenthal, J. Phys. Chem. A, 2014, 118, 10639-10648.

9 R. Lincoln, L. Kohler, S. Monro, H. Yin, M. Stephenson, R. Zong, A. Chouai, C. Dorsey, R. Hennigar, R. P. Thummel and S. A. McFarland, J. Am. Chem. Soc., 2013, 135, 17161-17175.

10 S. O. McDonnell, M. J. Hall, L. T. Allen, A. Byrne, W. M. Gallagher and D. F. O'Shea, J. Am. Chem. Soc., 2005, 127, 1636016361.

11 G. di Prisco, S. G. Condo, M. Tamburrini and B. Giardina, Trends Biochem. Sci., 1991, 16, 471-474.

12 Q. Zhao, F. Li and C. Huang, Chem. Soc. Rev., 2010, 39, 30073030.

13 J. Ma, J.-Y. Chen, M. Idowu and T. Nyokong, J. Phys. Chem. B, 2008, 112, 4465-4469.

14 E. J. McLaurin, A. B. Greytak, M. G. Bawendi and D. G. Nocera, J. Am. Chem. Soc., 2009, 131, 12994-13001.

15 R. Xu, Y. Wang, X. Duan, K. Lu, D. Micheroni, A. Hu and W. Lin, J. Am. Chem. Soc., 2016, 138, 2158-2161. 
16 X.-Y. Dong, Y. Si, J.-S. Yang, C. Zhang, Z. Han, P. Luo, Z.-Y. Wang, S.-Q. Zang and T. C. W. Mak, Nat. Commun., 2020, 11, 3678.

17 X. Wang, H. H. Gorris, J. A. Stolwijk, R. J. Meier, D. B. M. Groegel, J. Wegener and O. S. Wolfbeis, Chem. Sci., 2011, 2, 901906.

18 H. Zhang and Z. Zhang, Sensors, 2020, 20, 6175.

19 D. Kumar and C.-S. Chu, Sensors, 2021, 21, 4057.

20 K. S. Gkika, A. Kargaard, C. S. Burke, C. Dolan, A. Heise and T. E. Keyes, RSC Chem. Biol., 2021, 2, 1520-1533.

21 Q. Zhao, X. Zhou, T. Cao, K. Y. Zhang, L. Yang, S. Liu, H. Liang, H. Yang, F. Li and W. Huang, Chem. Sci., 2015, 6, 1825-1831.

22 E. Valdés, M. Cepeda-Plaza, G. Günther, A. Vega, R. Palacios, M. L. Gómez and N. Pizarro, Dyes Pigments, 2020, 172, 107787.

23 C. M. Tonge, N. R. Paisley, A. M. Polgar, K. Lix, W. R. Algar and Z. M. Hudson, ACS Appl. Mater. Interfaces, 2020, 12, 6525-6535. 24 T. Just Sørensen, A. M. Kenwright and S. Faulkner, Chem. Sci., 2015, 6, 2054-2059.

25 K. P. Anderson, M. A. Waddington, G. J. Balaich, J. M. Stauber, N. A. Bernier, J. R. Caram, P. I. Djurovich and A. M. Spokoyny, Dalton Trans., 2020, 49, 16245-16251.

26 L. Zang and H. Zhao, RSC Adv., 2020, 10, 32938-32945.

$27 \mathrm{H}$. Yersin, Highly efficient OLEDs with phosphorescent materials, Wiley-VCH, Weinheim, 2008.

28 E. Zysman-Colman, Ed., Iridium(III) in Optoelectronic and Photonics Applications, John Wiley \& Sons, Inc, Chichester, West Sussex, 2017.

29 H. Shi, X. Ma, Q. Zhao, B. Liu, Q. Qu, Z. An, Y. Zhao and W. Huang, Adv. Funct. Mater., 2014, 24, 4823-4830.

30 T. Yoshihara, S. Murayama and S. Tobita, Sensors, 2015, 15, 13503-13521.

31 K. S. Choung, K. Marroquin and T. S. Teets, Chem. Sci., 2019, 10, 5124-5132.

32 H. Na, M. Song and T. S. Teets, Chem. - Eur. J., 2019, 25, 48334842.

33 G. D. Sutton, K. S. Choung, K. Marroquin and T. S. Teets, Dalton Trans., 2020, 49, 13854-13861.

34 R. B. Moffett, J. Med. Chem., 1964, 7, 446-449.

35 V. Garg, G. Kodis, P. A. Liddell, Y. Terazono, T. A. Moore, A. L. Moore and D. Gust, J. Phys. Chem. B, 2013, 117, 11299-11308. 36 M. Nonoyama, Bull. Chem. Soc. Jpn., 1974, 47, 767-768.

37 A. Maity, J. C. Kölsch, H. Na and T. S. Teets, Dalton Trans., 2017, 46, 11757-11767.

38 S. Lamansky, P. Djurovich, D. Murphy, F. Abdel-Razzaq, H.-E. Lee, C. Adachi, P. E. Burrows, S. R. Forrest and M. E. Thompson, J. Am. Chem. Soc., 2001, 123, 4304-4312.

39 S. Takizawa, N. Ikuta, F. Zeng, S. Komaru, S. Sebata and S. Murata, Inorg. Chem., 2016, 55, 8723-8735.

40 Valeur, Molecular Fluorescence., Wiley VCH, Somerset, 2002. 41 N. J. Turro, V. Ramamurthy and J. C. Scaiano, Modern molecular photochemistry of organic molecules, University Science Books, Sausalito, Calif, 2010.

42 T. Sato, Y. Hamada, M. Sumikawa, S. Araki and H. Yamamoto, Ind. Eng. Chem. Res., 2014, 53, 19331-19337.

43 P. I. Djurovich, D. Murphy, M. E. Thompson, B. Hernandez, R. Gao, P. L. Hunt and M. Selke, Dalton Trans., 2007, 3763-3770.

44 P.-N. Lai, C. H. Brysacz, M. K. Alam, N. A. Ayoub, T. G. Gray, J. Bao and T. S. Teets, J. Am. Chem. Soc., 2018, 140, 10198-10207. 45 S. Yoon and T. S. Teets, Chem. Commun., 2021, 57, 19751988. 